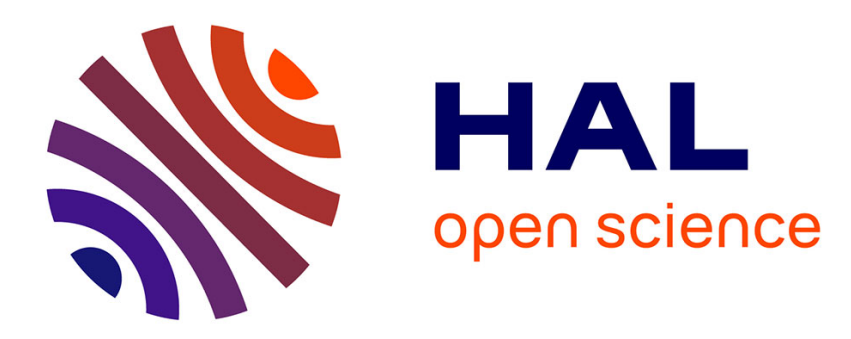

\title{
Numerical simulations of magnetic electron-impact ion source
}

\author{
Dmitry Ivanov, Bernard Bourdon
}

\section{To cite this version:}

Dmitry Ivanov, Bernard Bourdon. Numerical simulations of magnetic electron-impact ion source. International Journal of Mass Spectrometry, 2019, 10.1016/j.ijms.2019.05.005 . insu-02127249

\section{HAL Id: insu-02127249 \\ https://hal-insu.archives-ouvertes.fr/insu-02127249}

Submitted on 16 May 2019

HAL is a multi-disciplinary open access archive for the deposit and dissemination of scientific research documents, whether they are published or not. The documents may come from teaching and research institutions in France or abroad, or from public or private research centers.
L'archive ouverte pluridisciplinaire HAL, est destinée au dépôt et à la diffusion de documents scientifiques de niveau recherche, publiés ou non, émanant des établissements d'enseignement et de recherche français ou étrangers, des laboratoires publics ou privés. 


\section{Accepted Manuscript}

Numerical simulations of magnetic electron-impact ion source

Dmitry Ivanov, Bernard Bourdon

PII:

$$
\text { S1387-3806(19)30118-6 }
$$

DOI: $\quad$ https://doi.org/10.1016/j.jjms.2019.05.005

Reference: MASPEC 16151

To appear in: International Journal of Mass Spectrometry

Received Date: 18 March 2019

Revised Date: 6 May 2019

Accepted Date: 10 May 2019

Please cite this article as: D. Ivanov, B. Bourdon, Numerical simulations of magnetic electronimpact ion source, International Journal of Mass Spectrometry (2019), doi: https://doi.org/10.1016/ j.jjms.2019.05.005.

This is a PDF file of an unedited manuscript that has been accepted for publication. As a service to our customers we are providing this early version of the manuscript. The manuscript will undergo copyediting, typesetting, and review of the resulting proof before it is published in its final form. Please note that during the production process errors may be discovered which could affect the content, and all legal disclaimers that apply to the journal pertain. 


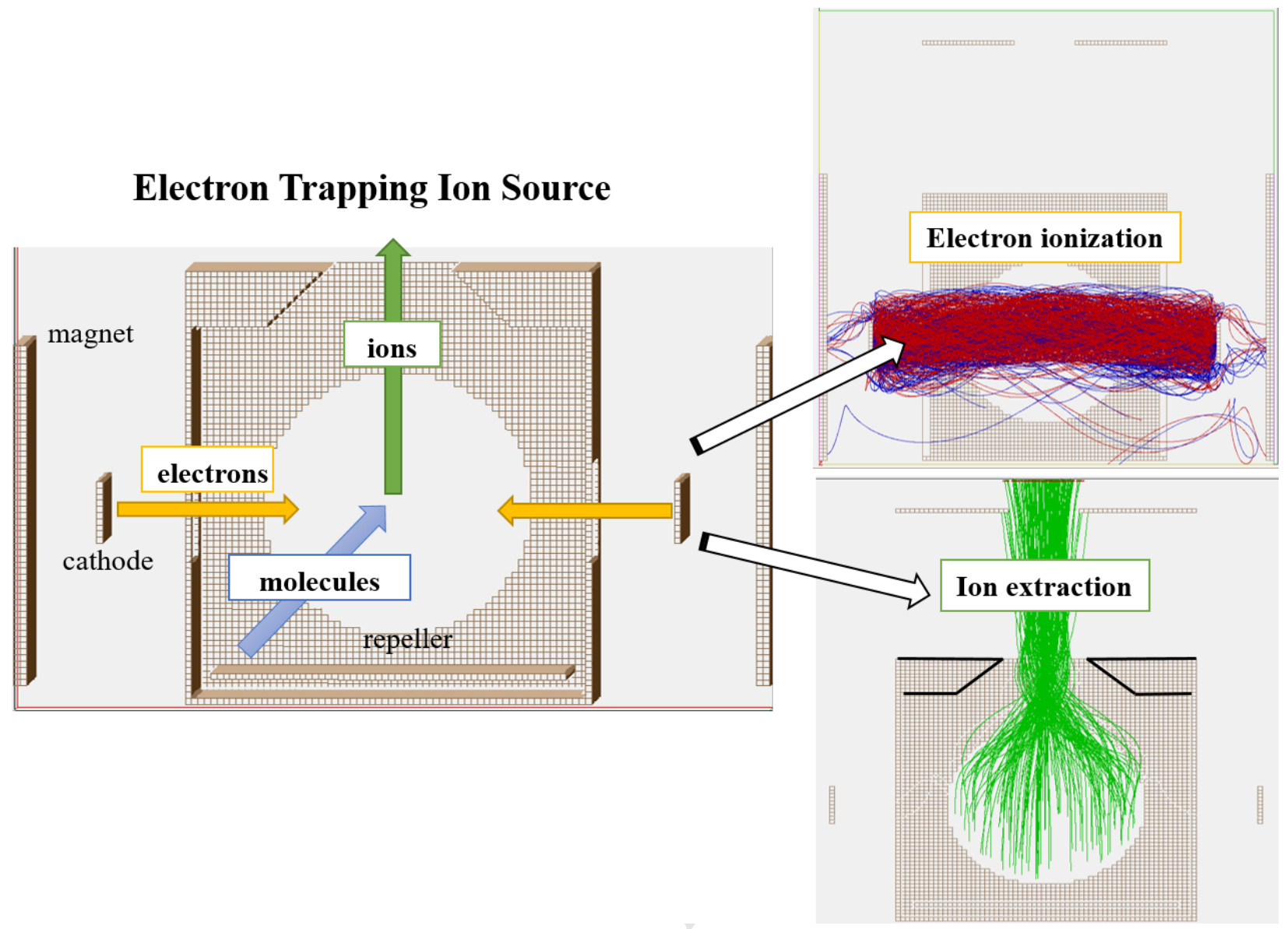




\title{
Numerical simulations of magnetic electron-impact ion source
}

\author{
Dmitry Ivanov, Bernard Bourdon * \\ Laboratoire de Géologie de Lyon, ENS Lyon, CNRS, UCBL, Université de Lyon, 46 allée d’Italie, 69364 Lyon, \\ France \\ * Corresponding author e-mail address: bernard.bourdon@ens-lyon.fr
}

\begin{abstract}
An electron impact ion source equipped with a magnet has been designed for enhancing the ionization efficiency of such ion sources with potential applications in Knudsen effusion mass spectrometry or electron impact sources used in gas mass spectrometry. This modernized device utilizes the trapping of electrons in parallel electrical and magnetic fields with the goal of maximizing the probability of impact between an electron and an atom or molecule to be ionized. The influence of magnetic and electrical fields on ions and electron trajectories inside the ion source was studied with numerical simulations. Based on the electron and ion trajectories we have modified the classical Nier-type ion source and found the optimal geometrical and potential parameters to provide better electron ionization and higher ion extraction efficiency. This design has the potential to enhance ionization efficiency by a factor of $\sim 15-20$ compared with existing designs.
\end{abstract}

Keywords: electron-impact ion source; computer simulations; electron ionization; ion extraction efficiency

\section{Introduction}

For highly precise isotope-ratio measurements, mass spectrometry is the most widely used technique [1]. Most of the instruments are based on a magnetic sector equipped with a multi collector data acquisition system. However, one of the key components of these instruments is the ion source whose operation determines the sensitivity and resolution parameters. One of the most commonly used ion sources in gas mass spectrometers is the Nier-type with ionization produced by electron-impact [2]. This type of source has also been used for Knudsen Effusion Mass Spectrometry where the sample is introduced in the ionization volume by a molecular flow stemming from a Knudsen cell [3]. The molecular flow is entering the ionization chamber where it is ionized by electrons emitted from a heated filament. Then, positive ions are accelerated by the electric fields of the extraction lenses with the help of a repeller field directed toward the extraction lens. The sensitivity of such ion source is largely determined by ionization efficiency which is directly related to the ion source configuration [4]. 
By using numerical simulations of ion and electron trajectories, it has become possible to estimate the ionization efficiencies of ion sources and investigate new promising approaches to significantly improve ion production and extraction into the mass analyzer. There are numerous parameters such as the geometry, the electrical and magnetic potentials, the nature of the vapor species and their ionization energies which can be modified and widely varied in all possible ranges by using 3-D computer simulations before its manufacturing. Among existing softwares, the SIMION program has successfully been used for developing a number of ion sources $[5,6,7$, 8].

Previous studies have shown the critical role of the electrical and magnetic field values on electron and ion trajectories for optimizing ionization and extraction efficiency [9, 10]. For example, in [11] the effect of the magnetic field on the sensitivity has been studied for two types of ion sources: the first one with electrons and ions having cross beam flow directions, while the second one had a co-axial electron and ion flows. In both cases, the ionization efficiency was enhanced by collimation of electrons into the chamber thanks to the effect of the magnetic field. A similar system was also studied with computer simulations in [12]. The authors showed an increase of the ion signal by a factor of up to 100 by a combination of collimation effect and helicoidal motion of electrons due to the applied magnetic field. Yet another design was proposal in $[13,14,15]$ where the molecular beam was co-axial with the electron beam and the path length of electrons was increased with a magnetic field. In this case, the specific goal was to reduce background at the expense of a reduced ion yield [13].

The aim of this work was to find the optimal parameters of electrical and magnetic fields which corresponds to the maximum of ionization efficiency for the ion source while keeping its size while allowing changes in geometry.

\section{Ion Source}

In this study, we have taken as a starting point the ionization chamber which is currently used in some Knudsen Effusion Mass Spectrometers as for example described in [3]. The geometry of the ion source is given in Fig.1 and the electron and ion trajectories were studied by numerical simulations with SIMION 8.1. The ion source is characterized by two filaments emitting electrons with a width of $1 \mathrm{~mm}$ and a height of about $1 \mathrm{~cm}$. There are two slits in front of the filaments used for accelerating the electron beam. The ionization chamber parameters is 
characterized by the following dimensions. The total ionization volume has an internal volume of $6 \times 6 \times 10 \mathrm{~mm}^{3}$. The slits in front of the electron emitting filaments (cathodes) have a width of 1.6 $\mathrm{mm}$. The distance between the cathodes and the slit is $1.2 \mathrm{~mm}$. The slit used for ion extraction has a variable width ranging between 4 to $2 \mathrm{~mm}$. Opposite to this extraction slit, there is a repeller plate used to enhance ion extraction. The diameter of the window for the inlet of the molecular beam is $\mathrm{d}=4.2 \mathrm{~mm}$.

A molecular beam $\left(N^{0}\right)$ is entering the ionization chamber 1 which is always at the ground potential in our study. The electrons $\bar{e}$ generated by the cathodes 2 enter the ionization chamber through slits 3 and bombard neutrals forming positive ions $N^{+}$. The ions are extracted from the ionization box through the extraction slit 5 by two lenses (located above the chamber) and by repeller potential 4. To improve ionization efficiency it is possible to modify the pathway of electrons by applying a magnetic field. For this purpose, two magnet plates 6 are placed behind the cathodes. All these species are moving in orthogonal directions: molecules are moving along the $\mathrm{OZ}$ axis, while the electrons and ions move along the $\mathrm{OX}$ and $\mathrm{OY}$ axis, respectively.

\section{Results of numerical simulations}

Electron and ion trajectories were studied by computer simulations for better understanding and optimizing the processes of ion production and ion extraction as a function of the applied electrical and magnetic fields. Charged particles are characterized by helical trajectories due to the combined effects of the electrical and magnetic field.

\subsection{Electron trajectories.}

For enhancing the probability of ionization by electron impact of atoms or molecules, two electron emitting cathodes were used. In principle, this should at least double the number of ions emitted per atom in the vapor comparing with a single cathode ion source. To further improve the electron ionization process, two magnets were placed behind cathodes in order to create a homogeneous magnetic field inside the chamber. It is a well known technique to modify electrons trajectories by increasing the length of their trajectories through the chamber, thereby increasing the probability of ionization. One should note however, that by applying a magnetic field, the electrons trajectories are modified while their kinetic energies remain constant. This is 
crucial as the efficiency of ionization by electron impact is strongly dependent on the electron energies [16]. Mathematically, this can be described by the following equations:

The forces acting on the electron can be written as:

$$
\boldsymbol{F}=q_{e}\left[\boldsymbol{E}(\boldsymbol{r}, t)+\frac{d \boldsymbol{r}}{d t}\right.
$$

where $r$ is a vector $(\mathrm{x}, \mathrm{y}, \mathrm{z})$ describing the position of the electron, $q_{e}$ is the charge of the electron, $E$ and $B$ are the electrical and magnetic fields, respectively. Thus, the equation of motion of electrons becomes:

$$
m_{e} \frac{d^{2} r}{d t^{2}}=q_{e}\left[\boldsymbol{E}(\boldsymbol{r}, t)+\frac{d \boldsymbol{r}}{d t} \wedge \boldsymbol{B}(\boldsymbol{r}, t)\right]
$$

where $m_{e}$ is the mass of an electron. The equation for the derivative of the kinetic energy is written as:

$$
\frac{m_{e}}{2} \frac{\mathrm{d}}{\mathrm{d} t}\left|\frac{\mathrm{d} \boldsymbol{r}}{\mathrm{d} t}\right|^{2}=q_{e} \boldsymbol{E}(\boldsymbol{r}, t) \cdot \frac{d \boldsymbol{r}}{d t}+q_{e}\left(\frac{d \boldsymbol{r}}{d t} \wedge \boldsymbol{B}(\boldsymbol{r}, t)\right) \cdot \frac{d \boldsymbol{r}}{d t}
$$

It becomes obvious based on this equation that the magnetic component of the kinetic energy is always equal to zero in this equation as $\mathrm{dr} / \mathrm{dt}$ is orthogonal to $\frac{d \boldsymbol{r}}{d t} \wedge \boldsymbol{B}$ and that the kinetic energy changes according to variations in the electrical field.

In order to describe the motion of electrons in the ionization chamber, we have considered an idealized view of the ion source by specifying three regions for electrons (see red arrows in Fig. 1). In region I, the electrons emitted from the left cathode are influenced by the combination of the electrical and magnetic fields: they are accelerated by the electrical field created by the potential between the cathode and ionization chamber walls. In this region, their trajectories are modified if they have an initial angular velocity component relative to direction Ox. In region II which is dominated by the magnetic field, the electrons move at constant kinetic energy as there is no accelerating voltage in that region. Then, they enter region III whose electrical field causes a deceleration of electrons due to the negative potential of the second cathode that ends up repelling the electrons back into the ionization chamber. This process is repeated until the electrons hit one of the surfaces: this means that the probability of an electron colliding with an atom or molecule of the beam is enhanced. This multi-pass effect is further 
enhanced by the gyromotion and collimation of electron trajectories. The system of equations that describes the electron trajectories can be derived by integrating equation (2):

$$
\left\{\begin{array}{l}
x(t)=V_{x}(0) t \pm \frac{e E t^{2}}{2 m_{e}} \\
y(t)=-\frac{V_{z}(0)}{\omega}+\frac{V_{z}(0)}{\omega} \cos \omega t+\frac{V_{y}(0)}{\omega} \sin \omega t \\
z(t)=\frac{V_{y}(0)}{\omega}-\frac{V_{y}(0)}{\omega} \cos \omega t+\frac{V_{z}(0)}{\omega} \sin \omega t
\end{array}\right.
$$

where $\omega$ is the electron cyclotron frequency:

$$
\omega=\frac{e B}{m_{e}}
$$

and $\mathrm{V}_{\mathrm{x}}, \mathrm{V}_{\mathrm{y}}, \mathrm{V}_{\mathrm{z}}$ are the $\mathrm{x}, \mathrm{y}, \mathrm{z}$ components of the electron velocity at the entrance of region II. To draw the trajectory of any electron in such a system, one needs to know the initial electron energy (depending on the cathode temperature), the cathode potentials, the magnetic field value and the angles of the trajectory when the electrons enter region II.

In addition to the effect of magnetic field, there is another possibility to enhance the probability of ionization. The electron entering slits can be exchanged with grids [17] for which we assume a transmission coefficient of 0.95 , corresponding to commercially available grids. This modification reduces the number of collisions of electrons with chamber walls and this extends the lifetime of electrons once they are emitted from the cathode. This becomes especially relevant since there are multiple passage of an electron inside the ionization chamber thanks to the influence of the magnetic field. This means that for each pass between the cathodes approximately $2 \times 5 \%$ of electrons are lost. Thus, it would take $\sim 6.6$ passes to lose half of the electrons on average. Altogether this suggests that the enhancement in ionization could be up to a significant factor (>7) relative to the case with no magnetic field. This reasoning can be confirmed with a slightly more quantitative approach. One can write a simple conservation equation for the number of electrons per unit of volume $n_{e}$ within the ionization volume:

$$
\frac{d n_{e}}{d t}=\varphi_{\text {in }}-\varphi_{\text {out }}
$$

Where $\varphi_{\text {in }}$ and $\varphi_{\text {out }}$ represents the average number of electrons produced in the ionization volume per unit of time and the number of electrons in the ionization volume lost per unit of time.

The number of electrons lost per unit of time can be expressed with the following equation: 


$$
\varphi_{\text {out }}=\iint_{S} 2 \bar{v} n_{e}(1-\sigma) d s=2 \bar{v} n_{e}(1-\sigma) S
$$

Where $S$ is the surface of the grids, $\bar{v}$ is the average velocity of electrons within the ionization volume, $\sigma$ is the void fraction of the grid (here 0.95 ). Thus, the mean density of electrons can simply be calculated assuming a steady-state situation as:

$$
n_{e}=\frac{\varphi_{\text {in }}}{2 \bar{v}(1-\sigma) S}
$$

In the case, when there is no magnetic field, the density of charges can be calculated simply as:

$$
n_{e}=\frac{\varphi_{i n}}{\bar{v} s}
$$

Hence, the enhancement in ionization due to the magnetic field is $1 /(2 *(1-\sigma))=10$.

An additional factor contributes to enhancing the ionization efficiency: our simulations show that the spiral electron trajectory is approximately $1.5-2$ times longer compared with the linear one without a magnetic field. As a consequence, the total increase in the ionization efficiency is around a factor of 15-20. It is also clear in this simulation that the electrons cover the whole volume of the ionization space providing a maximum ionization efficiency.

Figure 2 illustrates how the shape of potential lines is modified by introducing grids instead of slits. Note that in SIMION simulations, the ideal grids have infinitely small holes (there is no field penetration) and are perfectly transparent to electrons with a transmission of $100 \%$ and were at a potential of $0 \mathrm{~V}$. In these simulations, the cathode voltage is $-40 \mathrm{~V}$ while the extraction lenses are at $-400 \mathrm{~V}$ and ionization box and the repeller are at the ground $(0 \mathrm{~V})$. The introduction of grids produces more parallel potential lines, which is favorable for the extraction of ions. The difference in the number of extracted ions is $4 \%$ according to our simulations. Furthermore, the shapes of the potential lines limit the number of electrons lost to the walls as shown below.

Considering the potential impact of all the modifications described above, we first provide a simulation for 1 electron emitted from the center of the cathode in direction with the following angular coordinates as an example of a long electron trajectory: azimuth of $45^{\circ}$ and elevation of $22^{\circ}$. The initial kinetic energy of $0.215 \mathrm{eV}$ corresponds to thermoelectric emission from tungsten cathode at a temperature of $2500 \mathrm{~K}$. In this calculation, the cathode potentials were $-40 \mathrm{~V}$, and the extraction lenses were at $-400 \mathrm{~V}$. Supplementary Fig. S1 shows the electron trajectory under these conditions. In the absence of a magnetic field the electron has a linear trajectory (Supplementary Fig. S1a). The time of flight in this case is $0.0036 \mu$ s. When the 
magnetic field value at the center is $130 \mathrm{G}$ the electron has a helicoidal trajectory and multiple reflections take place (Supplementary Fig. S1b). The reflections are possible because the velocity component is not orthogonal to the filament surface where electrons are reflected and is therefore too low for the electron to impact the filament before being reflected. In this case, the calculated time of flight is $0.6907 \mu$ s. Thus, the ratio of the times of flight is $\operatorname{TOF}(127 \mathrm{G}) / \mathrm{TOF}(0 \mathrm{G})=192$. The simulation shows that the electron crossed the center region of ionization chamber 273 times (red dots shown in Supplementary Fig. S1b) before impacting a wall.

In the next step, we performed SIMION simulations using the source geometry shown in Supplementary Fig. S1 with the magnetic field taken as a free parameter. The system was simulated using $2 \times 50$ electrons emitted from $1 \mathrm{~mm}$ diameter circle at the surface of both cathodes. The initial velocities of the electrons was assumed to have a distribution within a cone of 1 degree half-angle. The results of these simulations are shown on Supplementary Fig. S2. The space charge has been shown to play a key role in the simulation of electron and ions trajectories [10]. This effect due to the accumulation of charges due to the trapping of electrons and it was taken into account using the POISSON module in SIMION. This module calculates the distribution of charges in a first stage and then recalculates its effect on trajectories iteratively using a particle-in-cell method. The input parameter for these calculations was assumed to be a $1 \mathrm{~mA}$ total current emitted from $1 \times 10 \mathrm{~mm}^{2}$ cathode. To simulate the effect of space charge, it is assumed that each electron carries a fraction of the total current of each cathode (1mA). In this case, the current carried by each electron is $20 \mu \mathrm{A}$. This is a classical approach describing the electron current with 'super-particles' that have a charge higher than that of true electrons. All other parameter were kept the same as mentioned above. The results obtained for various values of the magnetic field are shown in Fig. 3.

Overall, the effect of space charge is limited for the charge density obtained inside the ionization chamber (which is approximately a factor of 15-20 greater when using grids). The simulations (Fig. 3) show that the electrons have a slightly broader dispersion than in the case without space charge. By calculating the average time of flight (TOF) for electrons it is possible to quantitatively estimate the ionization probability depending on magnetic field values. In Fig. 4 the results of TOF calculations are shown for various values of the magnetic field between 0 and $350 \mathrm{G}$. The longest time of flight for electrons is obtained for $\mathrm{B}=127 \mathrm{G}$, which corresponds to the greatest probability of ionization. It is also possible to calculate the distribution of the TOF 
for this particular value of the magnetic field (Supplementary Fig. S3). The results show that the TOF follow more or less an exponential distribution. For each simulation, fifty electrons were generated from a $1 \mathrm{~mm}$ diameter circle for each cathode with $-40 \mathrm{~V}$ potential. The simulations were first run with no magnetic field to illustrate the influence of the electrical field on electrons trajectories. Using a weak magnetic fields $(<80 \mathrm{G})$ electron trajectories are more confined with a smaller angular dispersion in the XY plane. This leads to a higher electron density in the region of the molecular beam (shown by the circular section of the electron beam in the XY plane (Fig. 3). With a magnetic field $>100$ Gauss, there is a reflection of electrons between the two cathodes contributing to a much greater electron density. It was shown that the maximum ionization efficiency is obtained for a magnetic field equal to 127 Gauss.

Our SIMION simulations were also used to test the dependence of electron trajectories on the cathode potential. Various cathode voltages were applied while the magnetic field was kept constant at $\mathrm{B}=127$ Gauss (Fig. 5). The simulations clearly show that the ionization efficiency has no direct proportional relationship with the cathode potential. As the cathode potential increases, it can be seen that the electron trajectories do not remain stable and that instabilities leading to the loss of electrons to ionization chamber walls are observed. Such effect has been described for electron impact sources using magnet [16] but our simulations shows that this effect is not an artifact. If one estimates the ionization efficiency as a function of cathode potential, there are interleaving maximum and minimum in ionization efficiency. This effect can potentially be eliminated by using an electromagnet system instead of a permanent magnet. With an electromagnet it is also possible for the operator to switch it off during the recording of ionization efficiency curves [16], which means that no variation in ionization efficiency as a function of cathode potential will be seen. Furthermore, by varying the electromagnet potential it is then possible to adjust the maximum ion signal. As shown in [11], an electromagnet with fifty coils of pure iron at current $4 \mathrm{~A}$ would be sufficient to generate the magnetic field intensity of 160 G. Our simulation assumed a perfect and homogeneous magnetic field and a true electromagnet would certainly have fringe effects that should be examined in a real case.

Thus, our simulations show that there would be a considerable advantage in using an electromagnet rather than a permanent magnet, when the ionized molecules are characterized by an optimal value of electron impact energies that are not constant. In this case, there has to be a double optimization of the cathode potential and magnetic field: the magnetic field should be 
adjusted to the optimal value similarly to what is shown in Fig. 3 and this value is itself dependent on the optimal value of the cathode potential, specific to each molecule vapor species.

\subsection{Ion extraction}

\subsubsection{Extraction slits}

In order to extract ions from the ionization box, an extraction potential is applied in the direction of ion propagation. The ability to extract ion efficiently will depend on the shape of the potential lines inside the ionization volume and on the shape of the electrodes at the exit. In this study, we tested both the effect of the shape of the extraction slit, the potential of the repeller and the extraction voltage. For these parameters, we attempted to determine the optimal extraction yield by following the trajectories of individual ions and counting those that passed through the extraction slit. A more comprehensive simulation would require applying these simulations to a specific mass spectrometer with a defined lens stack in order to determine the transmission of the ion beam through the lens stack to the entrance slit of the mass spectrometer. Extraction efficiency coefficient $k_{\text {ext }}$ was calculated as a ratio of extracted ions $N_{\text {ext }}$ to initially generated ions $N_{\text {gen }}$ :

$$
k_{\text {ext }}=N_{\text {ext }} / N_{\text {gen }}
$$

The space charge effect of the ion beam was taken into account for extraction simulations. It was assumed 500 generated ions create an ion current of $10^{-9} \mathrm{~A}$ which is a typical value of ion currents in mass spectrometer experiments.

First, two shapes for the ion extraction slit were investigated. The extraction yields for a 'V-type' (see Fig. 6a) and a ' $\wedge$-type' shape (see Fig. 6b) were compared. Five hundred ions were generated within the ionization area inside the chamber. To extract the ions two plates located above the exit slit at a distance of $3 \mathrm{~mm}$ were used. The extraction potential was $-400 \mathrm{~V}$ and the repeller plate was set at ground potential. This corresponds to an electrical field of approximately $1.3 \mathrm{~V} / \mathrm{m}$ at the center of ionization chamber. Fig. 6 shows the ion trajectories and potential lines inside the chamber for different shapes of extraction slits. The orientation of the $\Lambda$-type slit (Fig. 6b) changes significantly the shape of potential lines near the exit slit. As a result it leads to a better focused ion beam and a better extraction. The simulations gave the following statistics for the extraction yields for $V$-type and $\Lambda$-type slits: $80 \%$ and $84 \%$ respectively. Thus, the $\Lambda$-type is a more efficient shape for ion extraction. Furthermore, the effect of the potential gradient in the 
center of the ionization volume was also tested and it was found that the maximum extraction was obtained for an electrical field of $1.6 \mathrm{~V} / \mathrm{mm}$ (Supplementary Fig. S4) for a repeller potential of $2 \mathrm{~V}$. This has implications for the energy dispersion of ions in the mass spectrometers since the region from which the ions are extracted will then define their energy dispersion.

\subsubsection{Extraction efficiency as a function of the repeller potential}

In a classical Nier-type source, the ionization chamber is at ground potential and the repeller plate is set at a positive potential. In this study, we investigated a wider range of potentials for the repeller plate. To obtain the optimal parameters, we varied the repeller voltage from $-4 \mathrm{~V}$ to $+10 \mathrm{~V}$ and calculated the extraction efficiency for each value using the method described above. Extraction potential was fixed at $-400 \mathrm{~V}$ (corresponding to a field of $1.6 \mathrm{~V} / \mathrm{mm}$ at the center of the ionization volume). The results of these simulations are presented in Fig. 7. For repeller potentials ranging between $2 \mathrm{~V}$ to $+10 \mathrm{~V}$, the extraction yield decreases. The highest extraction efficiency was obtained around $2 \mathrm{~V}$. Thus, the highest extraction efficiency is obtained when the repeller and ionization box potential differences are low. This fact is consistent with the results described by Ahn and Park [18]. Based on their computer simulations, these authors modified a Nier-type ion source by setting the same voltage $(+30 \mathrm{~V})$ on both the ionization chamber and repeller and also obtained better ion extraction. The shape of potential lines plays an essential role in controlling the extraction yields as shown in Fig. 8. If the repeller is at a high positive voltage, the generated ions are accelerated to a larger kinetic energy and this means that the angular dispersion of some ions cannot be fully corrected to go through the exit slit of the ionization chamber. Thus, these ions are lost by collisions with the walls of the chamber or of the extraction lenses. The ion beam becomes more spread by increasing the repeller potential (see the ion trajectories in Fig. 8). In contrast, when the repeller potential is only slightly positive, the ions are less accelerated compared with previous cases, which results in a better focusing into the exit slit. However, the better focusing could in fact be explained by the shapes of potential lines. The ions start moving perpendicularly to the potential lines inside the chamber and then there is a crossing point of their trajectories near the edge of the ionization volume. The extraction efficiency depends on the angles between ion trajectories and extraction direction axis (OY) before the crossing point. In the case of positive voltages of the repeller the potential lines are more flattened in the direction of the axis of extraction. This leads to defocusing of the ion beam 
with a greater dispersion at the extraction slit. In contrast, for slightly positive repeller potential the ion beam becomes more focused due to more elongate shapes for potential lines in the ion extraction direction.

\subsubsection{Effect of the magnetic field influence on the ion trajectories and extraction}

When the ions are generated inside the ionization volume and are accelerated towards extraction lenses, they are submitted to the Lorentz force which can modify their trajectories. As the ions masses are much larger than those of electrons, this influence is much weaker compared with electrons. Nevertheless, it is worth checking that the mass discrimination effect due to source magnetic field is not significant compared with other effects of mass fractionation. For example, Caro et al. [19] had shown that the source magnet used to deflect electrons in a thermal ionization mass spectrometer could have an influence that needed to be corrected for high precision $\mathrm{Nd}$ isotope measurements at the ppm level. Here, we have examined whether the source magnet could produce a significant mass fractionation using a simulation of ion extraction with $\mathrm{Mg}$ isotopes. We generated 3 types of ions, ${ }^{24} \mathrm{Mg}^{+},{ }^{25} \mathrm{Mg}^{+},{ }^{26} \mathrm{Mg}^{+}$from the same starting point and had them flying with an extraction field of $-400 \mathrm{~V}$ at a $3 \mathrm{~mm}$ distance from the ionization box and with a magnetic field of 170 Gauss which is close to the maximum of ionization probability values. It was found that there was no significant difference in their position after extraction. The maximum spatial deviation between the isotopes 24 and 26 was 3 microns, compared with the width of the extraction slit of $1.5 \mathrm{~mm}$. In order to estimate the possible differences in extraction efficiencies, the extraction of $5 \times 500$ ions of each isotope was simulated. All the generated ions were properly focused into the extraction slit and there was no measurable effects on the ion trajectories. The influence of magnetic field on ions became observable when its value was 10 times higher. The maximum deviation was 50 microns (1\% difference in extraction efficiency for the ${ }^{24} \mathrm{Mg}^{+}$and ${ }^{26} \mathrm{Mg}^{+}$ions) at 1630 Gauss of magnetic field, which is far from the optimal value as shown in the previous section.

\section{Conclusions}

This study was focused on numerical simulations of an electron-impact ion source for specific applications in the field of Knudsen Effusion Mass Spectrometry with the goal of optimizing a number of parameters for the source design. Compared with existing electron 
impact sources, the results of our simulations show that by using a configuration with two filament source facing each other (instead of a trap), it is possible to produce a trap for electrons that greatly enhances the ionization yield of the source. This effect has been succinctly described in the literature, however our more comprehensive simulations permit to identify the key parameters for this type of source. In particular, it was found that there is an optimal value of the magnetic field for each cathode voltage, which means that for optimizing the electron energy for a given molecule, a new feature of this source is that the magnetic field has to be tuned for best ionization yield. This suggests that using a tunable electro-magnet would be better for this type of source. Our simulations also show that using grids instead of slits in front of the electron emitting filaments results in a longer confinement of the electrons in the trap. Altogether this configuration results in an increase of the ionization yield by a factor of $\sim 15-20$ compared to the case with a single filament.

In a second step, the conditions of ion extraction were also explored and it was found that the shape of the exit slit for the ions can be optimized using a $\Lambda$-shape instead of a $V$-shape, which was classically used. Next, our simulations showed that the potential of the repeller has to be slightly positive to obtain the best extraction yield. Last, our results also show that the value of the maximum magnetic field needs to be checked to avoid mass discrimination of ions during their extraction to the mass analyzer but that the optimal value should not cause a mass discrimination $<1 \%$ o for magnesium isotopes.

Overall, the proposed design based on this electron trap should greatly enhance electron impact source with applications in noble gas mass spectrometry, Knudsen effusion mass spectrometer or gas source mass spectrometers used for isotope ratio measurements.

(Video files showing an example of these simulations from different view axis are available in on-line version).

\section{Acknowledgments}

The authors acknowledge the financial support of ERC funded project COSMOKEMS \# 694819. We thank Christian Chatillon, Johannes Schwieters and Gerhard Jung for fruitful discussions. We thank two anonymous reviewers who greatly helped improve the manuscript. 
Fig. 1. Electron-impact ion source model designed for KEMS analysis. The ionization volume has a symmetric configuration: 1 - ionization chamber; 2 - cathodes; 3 - electron passing slits; 4 - repeller; 5 - ion extraction slit; 6 - magnet plates. Electron motion in parallel electric and magnetic fields is shown by red arrows. For the electron motion from left to right: I - zone of magnetic and electric field (acceleration for electrons moving from left to right) influence; II magnetic field only; III - zone of magnetic and electric field (deceleration) influence.

Fig. 2. Potential contours (red lines) and gradient contours (blue lines) for ionization chamber with slits (a) and grids (b) for electrons. The approximate differences between potential lines are: at the bottom of the box $0.1 \mathrm{~V}$, at the center $0.2 \mathrm{~V}$, above the box $12.6 \mathrm{~V}$. The potential lines diagram highlights the more regular contours in the case of grids, which favors a better ion extraction.

Fig. 3. Electron trajectories in the ionization chamber as a function of the magnetic field, with the effect of space charge due to electron trapping.

Fig. 4. Average electron time of flight as a function of the magnetic field. Cathode voltage was set at $-40 \mathrm{~V}$ in this simulation. Solid line corresponds to calculations including the effect of space charge. Dashed line shows the electron time of flight in the absence of space charge. The value B $=127 \mathrm{G}$ corresponds to the greatest probability of ionization.

Fig. 5. Number of crossings of the center of ionization chamber by electrons as a function of cathodes potential with a magnetic field equal to $127 \mathrm{G}$. This parameter should be proportional to the density of charge in the ionization volume and can therefore be used as a proxy for the ionization efficiency. Solid line corresponds to calculations with the effect of space charge. Dashed line shows the results without space charge.

Fig. 6. Ion extraction with different slit shapes: $\mathbf{a}-\mathrm{V}$-type; $\mathbf{b}-\wedge$-type. $\wedge$-type slit changes the potential lines shape near the edge area, making the ion beam more focused and giving $4 \%$ difference in the extraction yields.

Fig. 7. Extraction efficiency as a function of the repeller voltage for a $-400 \mathrm{~V}$ extraction potential. The maximum extraction efficiency corresponds to $2 \mathrm{~V}$ for the repeller.

Fig. 8. Ion trajectories and potential lines for different repeller voltages. The ion extraction is better for low negative values of the repeller plate compared with positive values due to the more elongated potential lines in the extraction direction. 


\section{Supplementary materials}

Supplementary Fig. S1. Electron trajectories from the cathode center in parallel electrical and magnetic fields: (a) $B=0 \mathrm{G}$; (b) $B=127 \mathrm{G}$.

Supplementary Fig. S2. Electron trajectories in the ionization chamber as a function of the magnetic field, without the effect of space charge.

Supplementary Fig. S3. Electron time of flight distribution for a magnetic field, B = $127 \mathrm{G}$. Shaded columns - space charge calculations; unfilled - without space charge effect. The vertical red lines mark the average values of the TOF: solid line is for a case with space charge and the dashed one is in the absence of space charge. The inclusion of space charge results in a shorter residence time, yielding a lower ionization efficiency.

Supplementary Fig. S4. Ion extraction efficiency as a function of the electric potential gradient at the center of ionization chamber. 


\section{References}

[1] I.T. Platzner, K. Habfast, A.J. Walder, A. Goetz, Modern isotope ratio mass spectrometry, Chichester; New York: J. Wiley (1997). 530 p. ISBN: 978-0-471-97416-1

[2] A.O.C. Nier, A Mass Spectrometer for Isotope and Gas Analysis, Rev. Sci. Instrum. 18 (1947). 398-411. doi: 10.1063/1.1740961

[3] E.H. Copland, N.S. Jacobson, Measuring thermodynamic properties of metals and alloys with Knudsen Effusion mass spectrometry, NASA Report, NASA/TP-2010-216795, E-17245 (2010).

[4] K. Kuhnke, K. Kern, R. David, G. Comsa, High efficiency molecular beam ionization detector with short ionization region, Rev. Sci. Instrum. 65 (1994) 3458 - 3465 . doi: $10.1063 / 1.1144523$

[5] D.A. Dahl, SIMION for the personal computer in reflection, Int. J. Mass Spectrom. 200 (2000) 3-25. doi: 10.1016/S1387-3806(00)00305-5

[6] A. Basak, R.K. Bhati, V.K. Yadav, E. Ravisankar, T.K.Saha, V.Nataraju, S.C. Gadkari, An improved ion source for thermal ionization mass spectrometer, Int. J. Mass Spectrom. 423 (2017) 33-38. doi: 10.1016/j.ijms.2017.10.006

[7] C. Maden, A. Trinquier, A.L. Fauré, A. Hubert, F. Pointurier, J. Rickli, B. Bourdon, Design of a prototype thermal ionization cavity source intended for isotope ratio analysis, Int. J. Mass Spectrom. 434 (2018) 70-80. doi: 10.1016/j.ijms.2018.09.006

[8] A.D. Appelhans, J.E. Olson, D.A. Dahl, M.B. Ward. High-Efficiency Noble Gas Electron Impact Ion Source for Isotope Separation, INL/EXT-16-39503. (2016)

[9] H.W. Werner, A study of a mass spectrometer ion source by means of computer-calculated ion trajectories, J. Phys. E: Sci. Instrum. 7 (1974) 115-121. doi: 10.1088/0022-3735/7/2/013

[10] A.R. Alderwick, A.P. Jardine, H. Hedgeland, D.A. MacLaren, W. Allison, J. Ellis, Simulation and analysis of solenoidal ion sources, Rev. Sci. Instrum. 79 (2008) 123301 123301-9. doi: 10.1063/1.3030858

[11] C.J. Park, J.R. Ahn, Effect of magnetic field in electron-impact ion sources and simulation of electron trajectories, Rev. Sci. Instrum. 77 (2006) 085107-085107-5. doi: 10.1063/1.2336756

[12] O. Hadjar, W.K. Fowler, High-efficiency cross-beam magnetic electron-impact source for improved miniature Mattauch-Herzog mass spectrometer performance, Rev. Sci. Instrum. 83 (2012) 064101-064101-6. doi: 10.1063/1.4729115 
[13] M. DeKieviet, D. Dubbers, M. Klein, U. Pieles, C. Schmidt, Design and performance of a highly efficient mass spectrometer for molecular beams, Rev. Sci. Instrum. 71 (2000) 20152018. doi: $10.1063 / 1.1150570$

[14] A.V. Kalinin, L.Yu. Rusin, J.P. Toennies, Ion source with longitudinal ionization of a molecular beam by an electron beam in a magnetic field, Instrum. Exp. Tech. 49 (2006) 709713. doi: 10.1134/S0020441206050186

[15] F. Muntean, Magnetically assisted electron impact ion source for mass spectrometry, US Patent 9,721,777 B1 (2017)

[16] J. Drowart, C. Chatillon, J. Hastie, and D. Bonnell, High-Temperature Mass Spectrometry: Instrumental Techniques, Ionization Cross- Sections, Pressure Measurements, and Thermodynamic Data (IUPAC Technical Report), Pure Appl. Chem. 77 (2005) 683-737. doi: $10.1351 /$ pac200577040683

[17] R. Weiss, Molecular Beam Electron Bombardment Detector, Rev. Sci. Instrum. 32 (1961) 397 - 401. doi: 10.1063/1.1717386

[18] J.R. Ahn, C.J. Park, Computer simulation of electron and ion trajectories in electronimpaction sources of a quadrupole mass spectrometer, Nucl. Instrum. Methods Phys. Res A 645 (2011) 345-349. doi:10.1016/j.nima.2011.01.165

[19] G. Caro, B. Bourdon, J.-L. Birck, S. Moorbath, High-precision ${ }^{142} \mathrm{Nd} /{ }^{144} \mathrm{Nd}$ measurements in terrestrial rocks: Constraints on the early differentiation of the Earth's mantle, Geochim. Cosmochim. Acta. 70 (2006) 164-191. doi:10.1016/j.gca.2005.08.015 


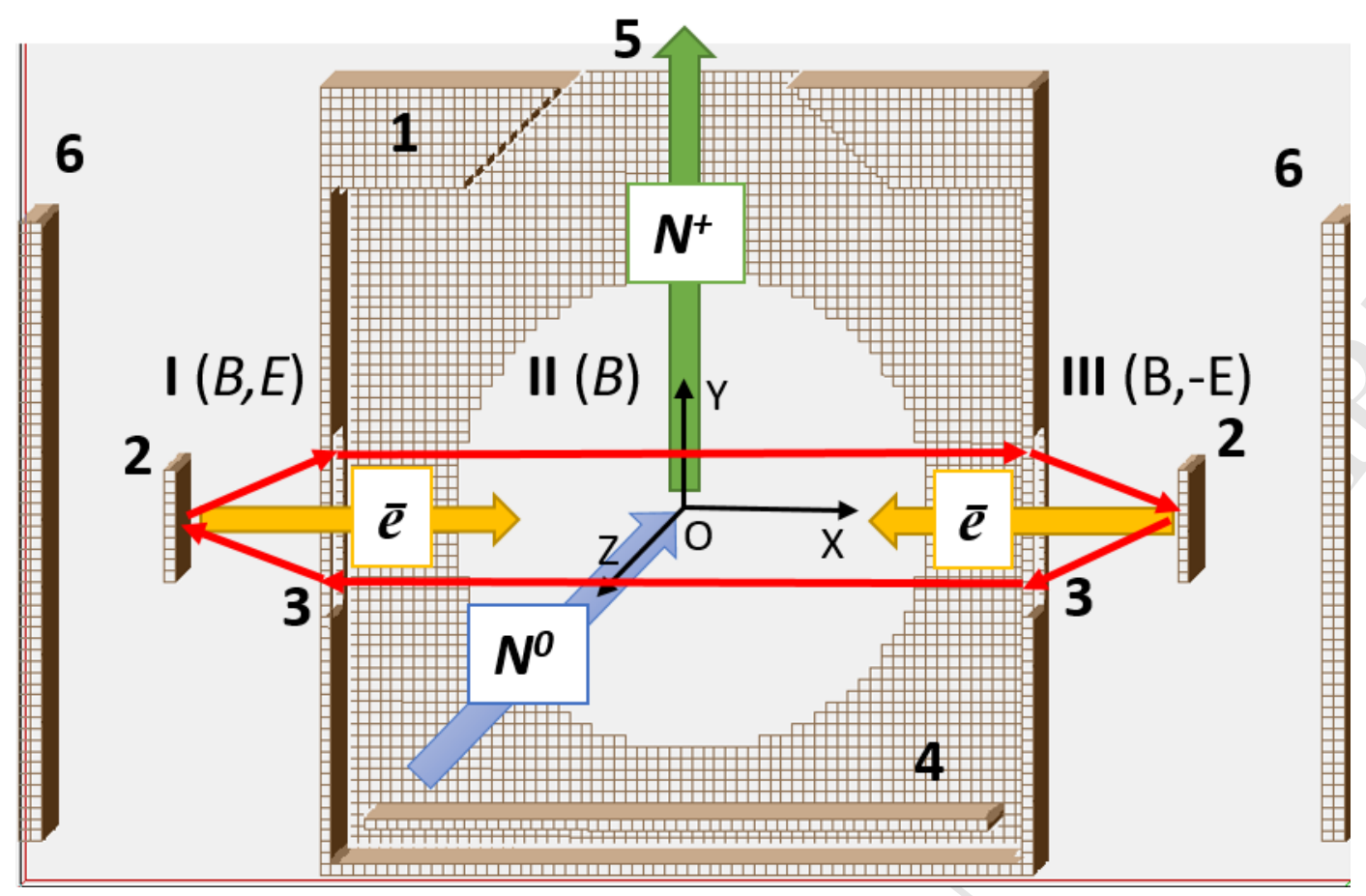



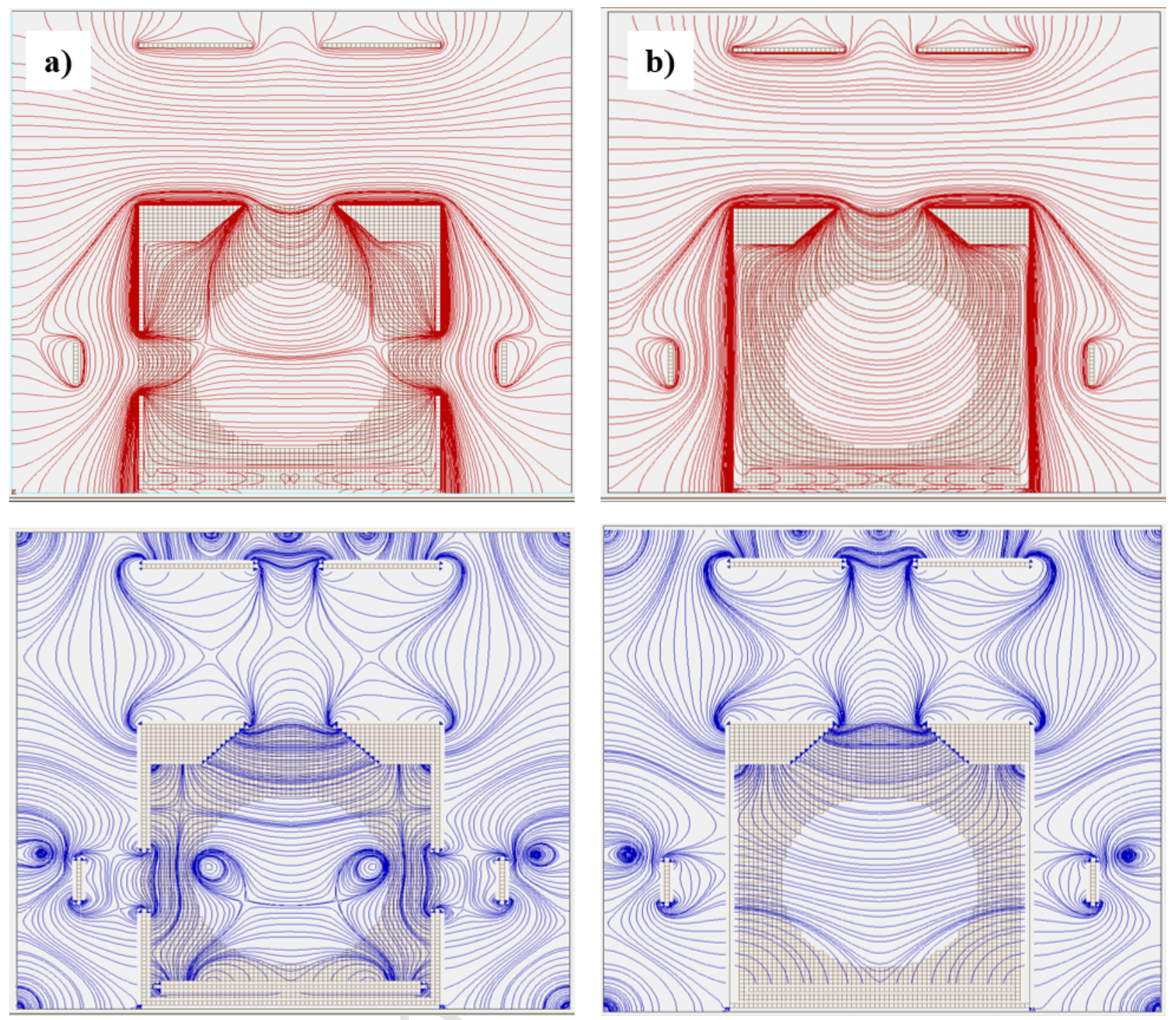

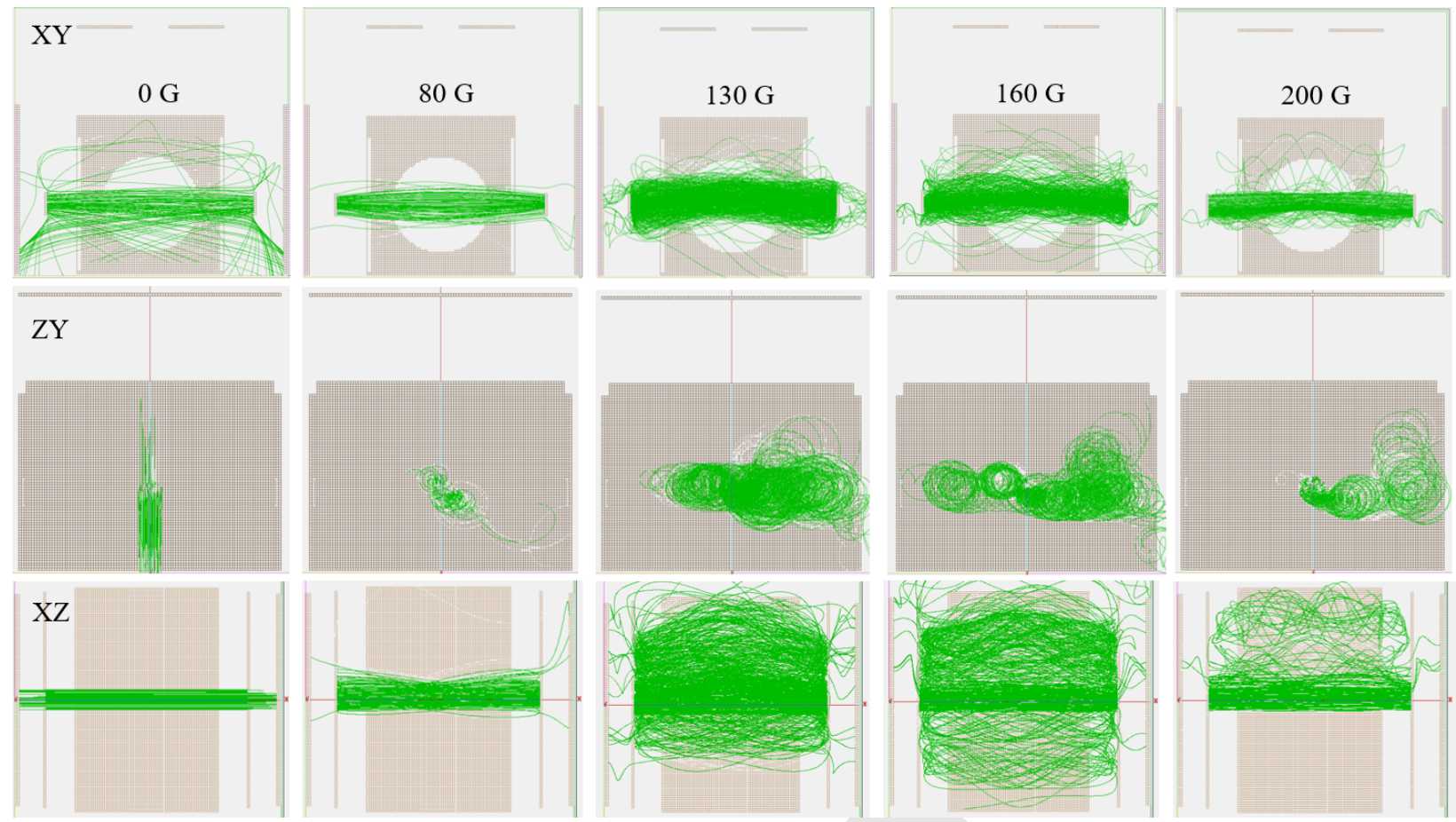


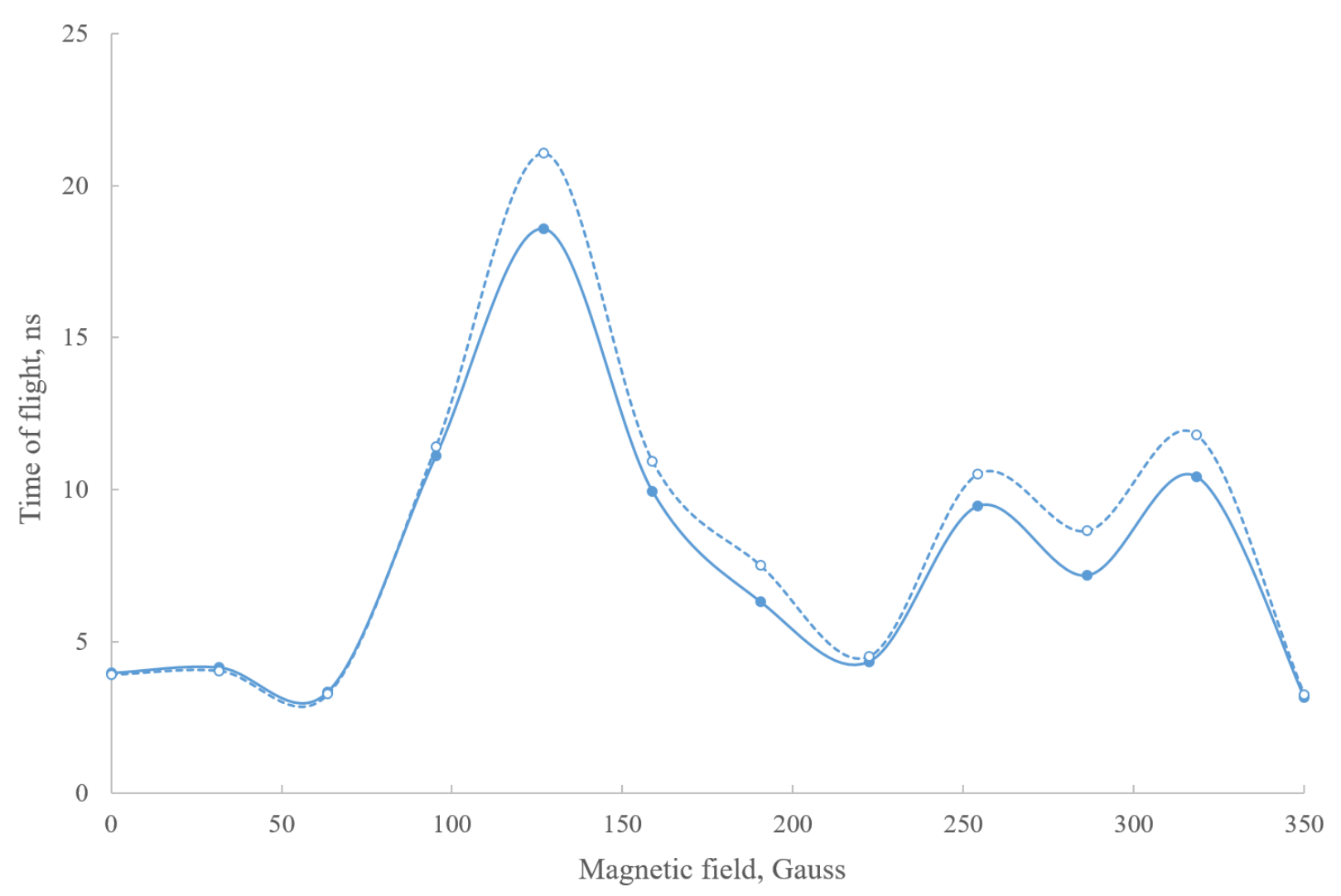




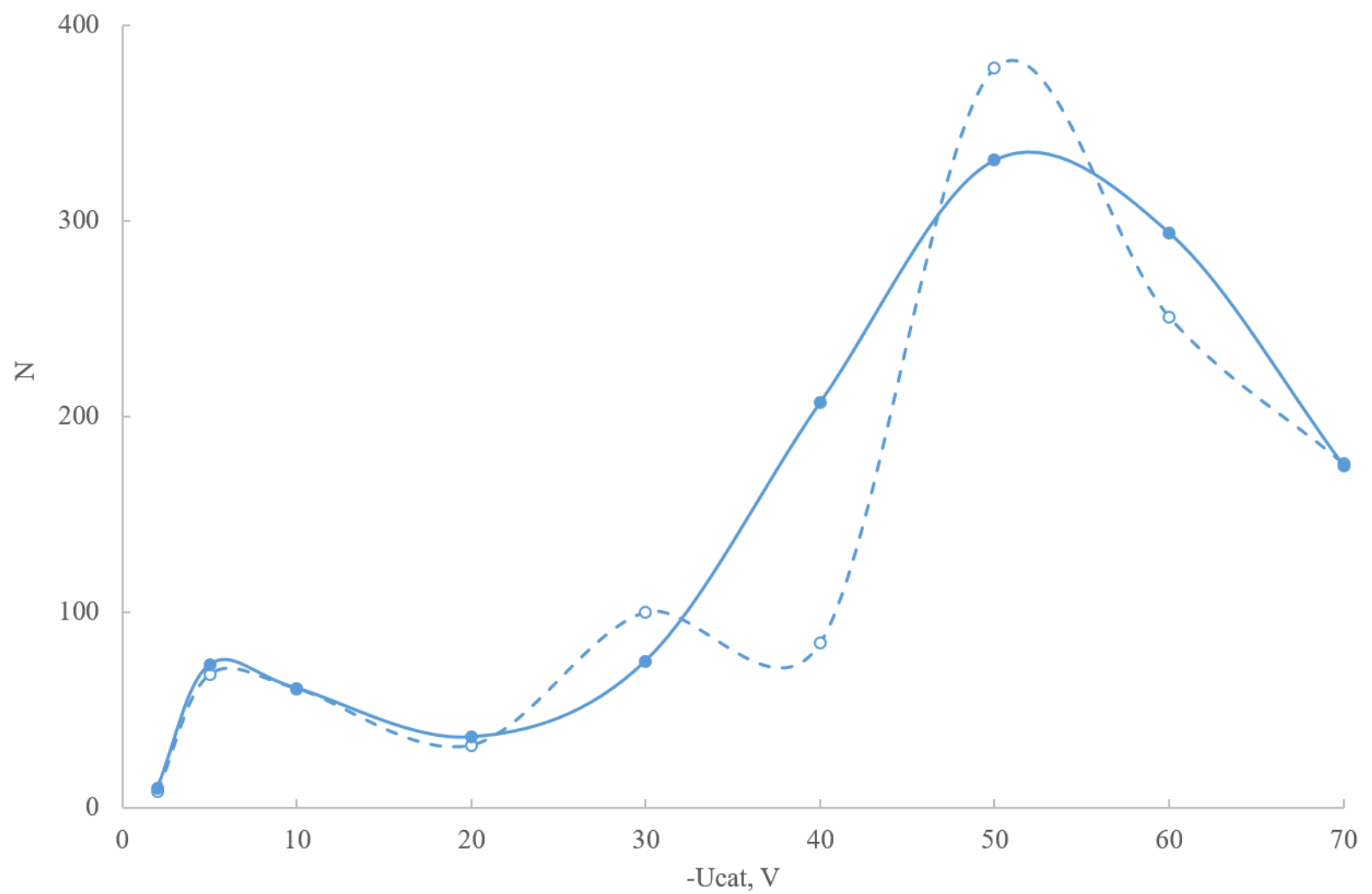




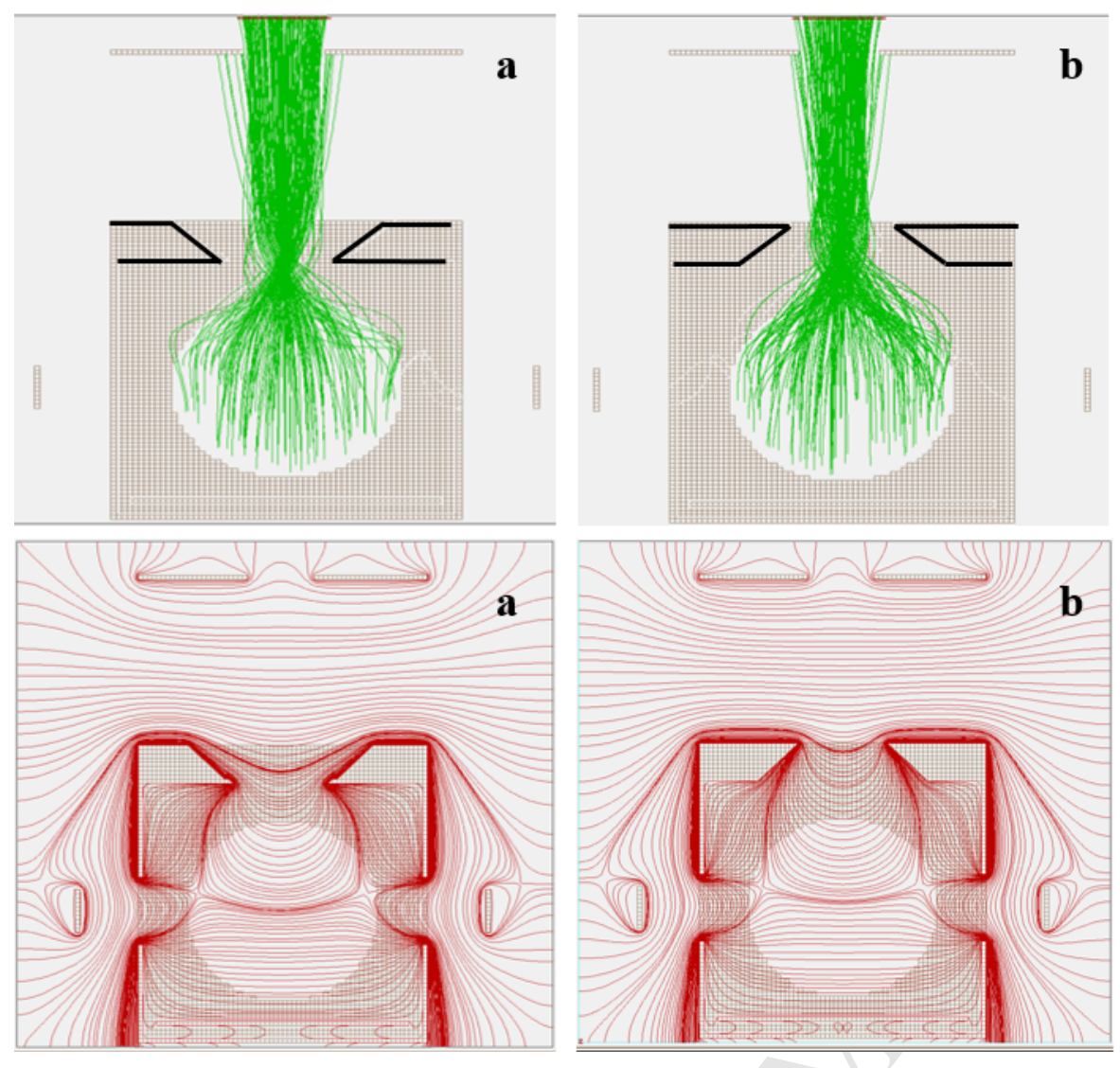




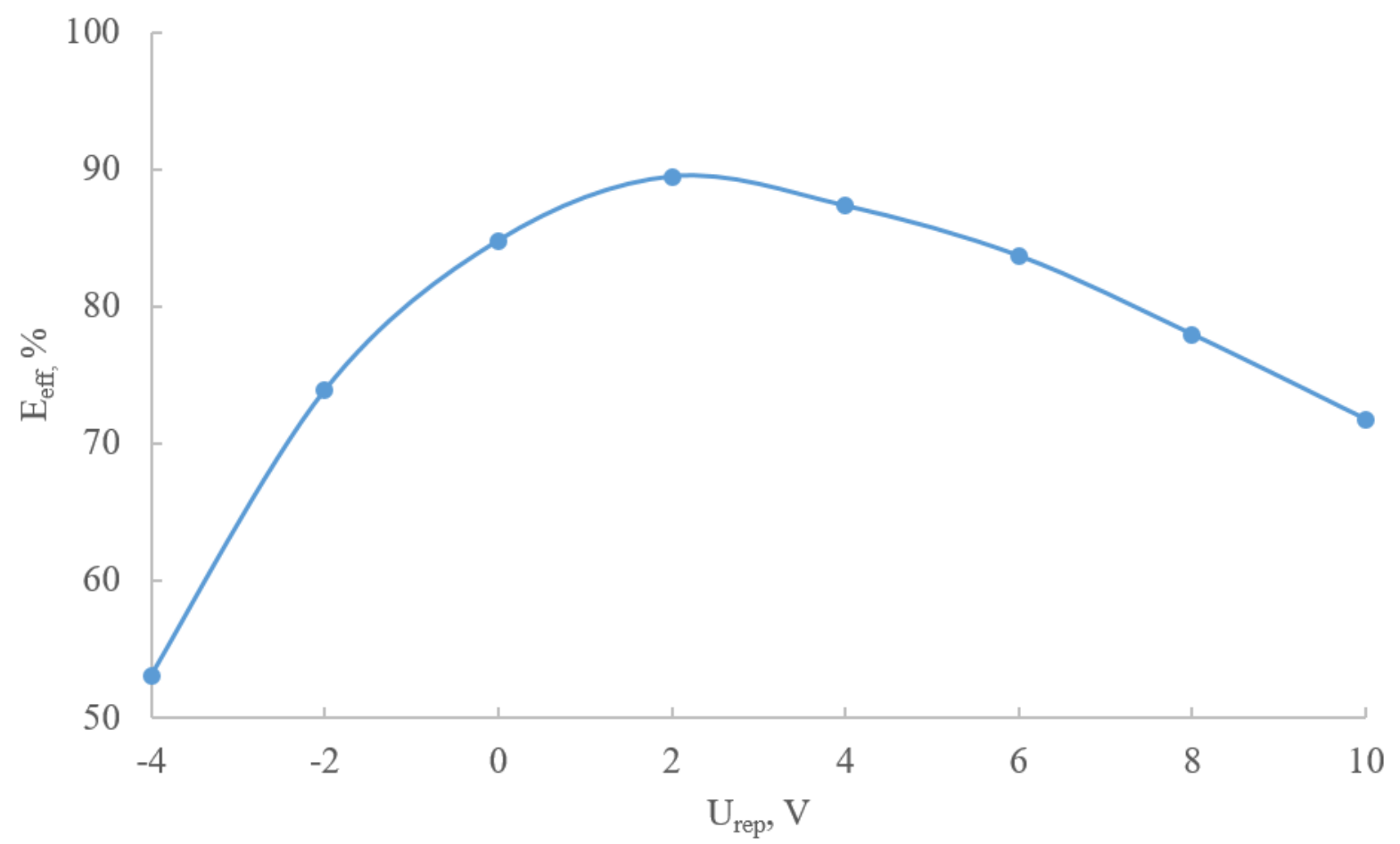



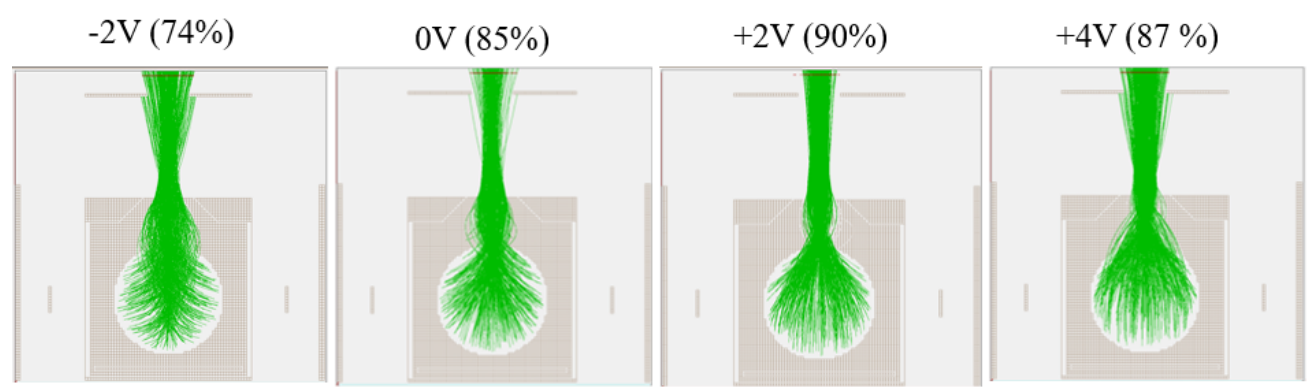

$+10 \mathrm{~V}(72 \%)$
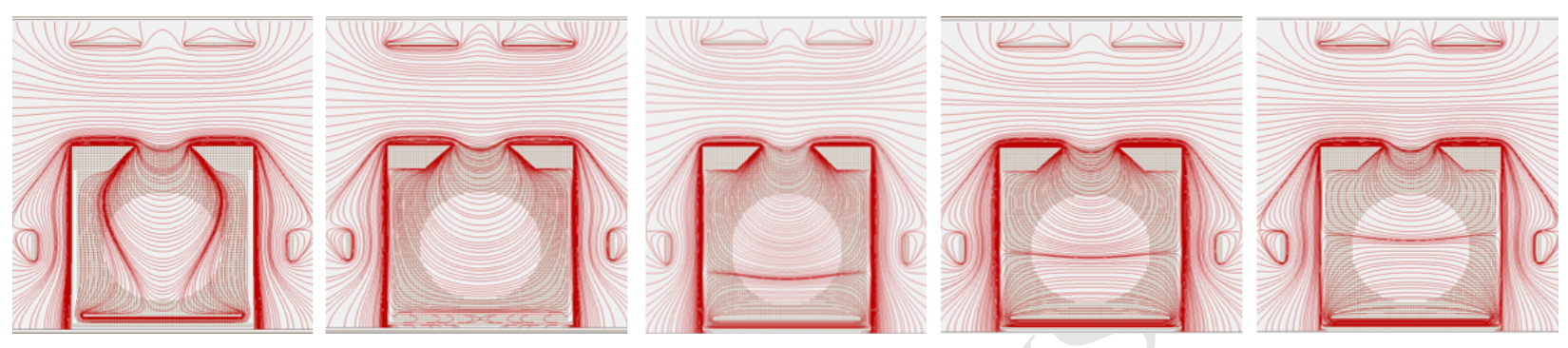


\section{Highlights}

A new magnetic electron-impact ion source for Knudsen effusion mass spectrometry has been designed.

The optimal geometrical parameters, electrical and magnetic fields magnitudes were determined by computer simulations for the maximum of ionization efficiency.

The effects of space charge and mass discrimination in the ion source have been analyzed. 